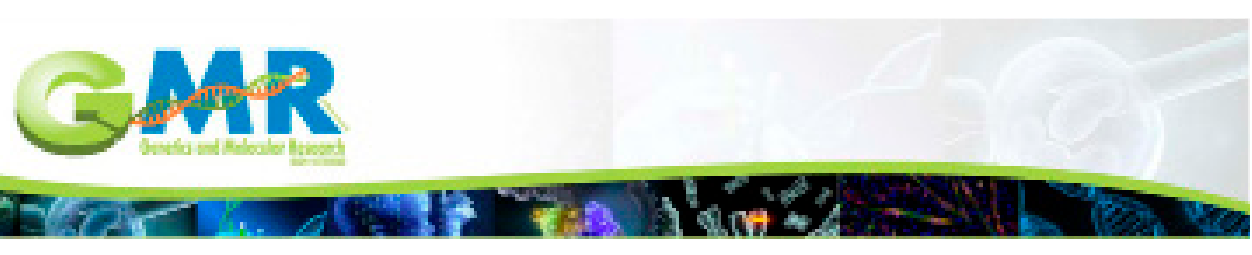

\title{
Analysis of the gene-protein interaction network in glioma
}

\author{
C. Zhou ${ }^{1 *}$, W.J. Teng ${ }^{2 *}$, J. Zhuang ${ }^{1}$, H.L. Liu ${ }^{3}$, S.F. Tang ${ }^{1}$, X.J. Cao ${ }^{4}$, B.N. Qin ${ }^{4}$, \\ C.C. Wang ${ }^{4}$ and C.G. Sun ${ }^{1}$
}

${ }^{1}$ Cancer Center, WeiFang Traditional Chinese Hospital, Weifang, Shandong, China ${ }^{2}$ Shangai Municipal Hospital of Traditional Chinese Medicine,

Affiliated to Shangai Univerity of Traditional Chinese Medicine, Shanghai, China

${ }^{3}$ Guang'anmen Hospital, China Academy of TCM, Beijing, China

${ }^{4}$ Shandong University of Chinese Traditional Medicine, Jinan, Shandong, China

${ }^{*}$ These authors contributed equally to this study.

Corresponding author: C.G. Sun

E-mail: zhongliuyike@163.com

Genet. Mol. Res. 14 (4): 14196-14206 (2015)

Received May 3, 2015

Accepted August 14, 2015

Published November 13, 2015

DOI http://dx.doi.org/10.4238/2015.November.13.3

ABSTRACT. Glioma is the most aggressive type of brain tumor. Great progress has been achieved in glioma treatment, but the protein-protein interaction networks underlining glioma are poorly understood. We identified the protein-protein interaction network for glioma based on gene expression and predicted biological pathways underlying the molecular complexes in the network. Genes involved in glioma were selected from the Online Mendelian Inheritance in Man (OMIM) database. A literature search was performed using the Agilent Literature Search plugin, and Cytoscape was used to establish a protein-protein interaction network. The molecular complexes in the network were detected using the Clusterviz plugin, and pathway enrichment of molecular complexes was performed using DAVID online. There were 378 glioma genes in the OMIM database. The protein-protein interaction network in glioma contained 1814 nodes, 6471 edges, and 8 molecular complexes. There were 17 pathways (false discovery rate $<1$ ), which were related to cytokinecytokine receptor interaction, Toll-like receptor signaling pathway, chemokine signaling pathway, oocyte meiosis, progesterone-mediated oocyte maturation, transmembrane transport of small molecules, metabolism of amino acids, and notch signaling pathway, among others. Our results provide a bioinformatic 
foundation for further studies of the mechanisms of glioma.

Key words: Molecular complexes; Protein-protein interaction networks; Glioma; Pathways

\section{INTRODUCTION}

Gliomas, which are the most aggressive type of brain tumor, show high morbidity, a high recurrence rate, and high mortality. Glioma accounts for approximately $30 \%$ of brain and central nervous system tumors and $80 \%$ of malignant brain tumors (Goodenberger and Jenkins, 2012; Shao et al., 2014). Survival of gliomas depends on the tumor type and malignancy grade (Constantin et al., 2012). According to World Health Organization standards, gliomas are classified into 4 malignant grades. Grade I-II gliomas can be treated with surgery and chemoradiotherapy, and are generally associated with a survival time of 5-10 years. The most lethal is grade IV glioblastoma, with a median survival of only 15 months (Wen and Kesari, 2008) because of the inefficacy of surgery and chemoradiotherapy. In addition, over $50 \%$ of low-grade gliomas undergo malignant transformation into high-grade gliomas within 5-10 years during recurrence (Dell'Albani, 2008). The prognosis of glioma, particularly high-grade (III-IV) glioma, is typically poor. Glioblastoma multiforme is the most predominant and most malignant form of glioma. Despite the high incidence of glioma, the etiology of this disease remains largely unknown.

Recently, the development of high-throughput experimental strategies has facilitated the study of characteristics underlying cancer progression. Several studies have investigated the gene expression signature in glioma patients (Ideker and Sharan, 2008; Zhao et al., 2008). Previous studies have mainly used regression or variance analysis to identify deregulated genes that may contribute to the glioma pathomechanism. However, these methods cannot address other array-specific factors, such as various background biological and environmental factors. Identifying the molecular characteristics of glioma patients may increase the understanding of the mechanism underlying glioma.

Because of the large number of targets involved in gliomas, the gene-protein network cannot be constructed using standard experiments. Numerous previous studies have examined gliomas, indicating that the gene-protein network can be constructed using a literature-mining method (Yang et al., 2009; Giacomelli and Covani, 2010). Construction through literature mining involves bioinformatics and computer science, among other fields, to sort and analyze existing data based on gene-protein interaction relationships to construct a regulation network of biological molecules in a cell. This method is important for identifying regulators and network-stable, therefore it has great application space (Strogatz, 2001; Pospisil et al., 2006).

To further examine glioma on the gene-protein network level, we used the human Mendel database to identify confirmed genes associated with gliomas, and then used Cytoscape application software to establish gliomas based on biological function gene-protein interaction networks. Subsequently, we determined topological properties and conducted modularity analysis of the network, and enriched the functional analysis and functional modules using DAVID software. We identified and analyzed key genes and signaling pathways in the network to predict the pathogenic site of the disease and the molecular mechanisms of gliomas.

\section{MATERIAL AND METHODS}

\section{Data acquisition}

On April 5, 2014 after searched "glioma" on the OMIM home page (http://www.ncbi.nlm. 
nih.gov/omim), gene information associated with gliomas was screened to remove duplicate genes (Amberger et al., 2009).

\section{Construction of gene-protein interaction networks}

Glioma-associated genes were searched in the Cytoscape 2.8.2 plug-in Agilent Literature Search 2.7.7 (USA Agilent Technologies, Santa Clara, CA, USA) and in Pubmed (Vailaya et al., 2005). False-positive interaction information was removed from the results. Next, gene/protein interaction relationships were read in Cytoscape 2.8.2 and visualized (Shannon et al., 2003).

\section{Network analysis}

The MCOMD algorithm in Cytoscape 2.8.2 web analytics plug-in Clusterviz of 1.2 was used for correlation analysis to construct biological networks (Saito et al., 2012). By analyzing the network structure, proteins were grouped to form molecular compounds in the entire network and were viewed in Cytoscape based on the correlation integral value. The areas with integral values higher than 3 were regarded as molecular compounds. The gene/protein names contained in the molecular compounds were submitted to The Database for Annotation, Visualization, and Integrated Discovery (Huang et al., 2009). Using the Kyoko Encyclopedia of Genes and Genomes (KEGG) Database, biological pathways involved in glioma heredity were identified.

\section{Main outcome measures}

Protein networks were constructed based on glioma-related genes, nodes (proteins) and edges (interaction between), molecular complexes in the network and its associated interaction points, and nodes (protein) and the edges (interaction between) to analyze the biological pathways involved in the molecular complexes.

\section{RESULTS}

\section{Glioma-related genes in OMIM}

Through OMIM database retrieval, we identified 378 genes related to glioma, as shown in Table 1.

\section{Protein interaction networks}

The 378 glioma-related genes identified were constructed into a network diagram with 1814 nodes (proteins) and 6471 edges. As shown in Figure 1, the triangles represent OMIM genetic disease-related proteins, while the diamonds represent proteins obtained from text mining.

\section{Network topology attribute analysis}

Network topology attribute analysis revealed that the connectivity of nodes in the network (the number of nodes in the network) had a descending distribution; as the edges connected to the node increased, the number of nodes decreased. Thus, the gene-protein interaction networks are scale-free networks (Burkard et al., 2010). We found that the degree of nodes in the network greater than or equal to 50 corresponded to a sharp reduction in the number of nodes (Figure 2). 


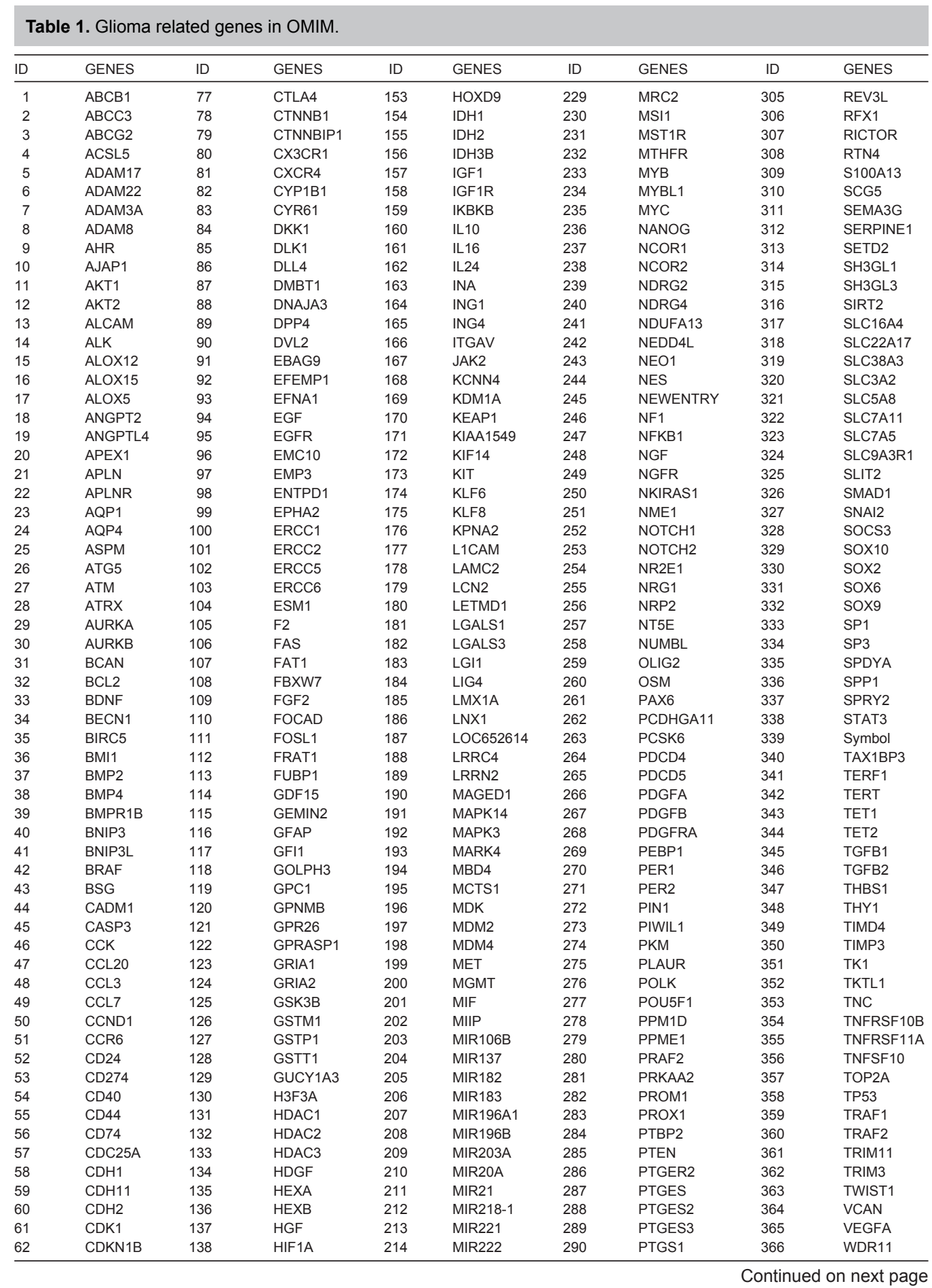




\begin{tabular}{|c|c|c|c|c|c|c|c|c|c|}
\hline ID & GENES & ID & GENES & ID & GENES & ID & GENES & ID & GENES \\
\hline 63 & CDKN2A & 139 & HJURP & 215 & MIR27B & 291 & PTGS2 & 367 & WNT1 \\
\hline 64 & CHEK2 & 140 & HK2 & 216 & MIR30A & 292 & PTK2 & 368 & WNT2 \\
\hline 65 & CHI3L1 & 141 & HLA-B & 217 & MIR335 & 293 & PTP4A3 & 369 & WNT5A \\
\hline 66 & CHN2 & 142 & HLA-C & 218 & MIR372 & 294 & PVR & 370 & WRN \\
\hline 67 & $\mathrm{ClC}$ & 143 & HLA-DQB1 & 219 & MIR375 & 295 & RAC2 & 371 & WT1 \\
\hline 68 & CLCN3 & 144 & HLA-DRB1 & 220 & MIR383 & 296 & RASL10A & 372 & WWTR1 \\
\hline 69 & CLIC1 & 145 & HLA-DRB3 & 221 & MIR410 & 297 & RASSF10 & 373 & XBP1 \\
\hline 70 & CNR1 & 146 & HMG20B & 222 & MIR452 & 298 & RB1 & 374 & XRCC1 \\
\hline 71 & CNR2 & 147 & HMGA1 & 223 & MIR483 & 299 & RBL2 & 375 & XRCC3 \\
\hline 72 & CNTFR & 148 & HMGN5 & 224 & MKI67 & 300 & RBP1 & 376 & XRCC4 \\
\hline 73 & COL18A1 & 149 & HNRNPA1 & 225 & MMP14 & 301 & RBPJ & 377 & YY1 \\
\hline 74 & CRABP2 & 150 & HNRNPA2B1 & 226 & MMP2 & 302 & RECQL & 378 & ZAR1 \\
\hline 75 & CSF2 & 151 & HNRNPH1 & 227 & MMP3 & 303 & REG4 & & \\
\hline 76 & CTGF & 152 & HOXA9 & 228 & MMP9 & 304 & RELA & & \\
\hline
\end{tabular}

Therefore, we regarded the nodes which connectivity is greater than or equal to 50 as key nodes (hub). Key nodes included akt1, tnfsf13, tp53, ephb2, pik3ca, mapk3, mapk14, il6, cdkn1a, vegfa, mapk8, stat3, egfr, myc, bcl2, cdkn2a, apc, ptgs2, pten, hcc, ccl2, and ervk2.
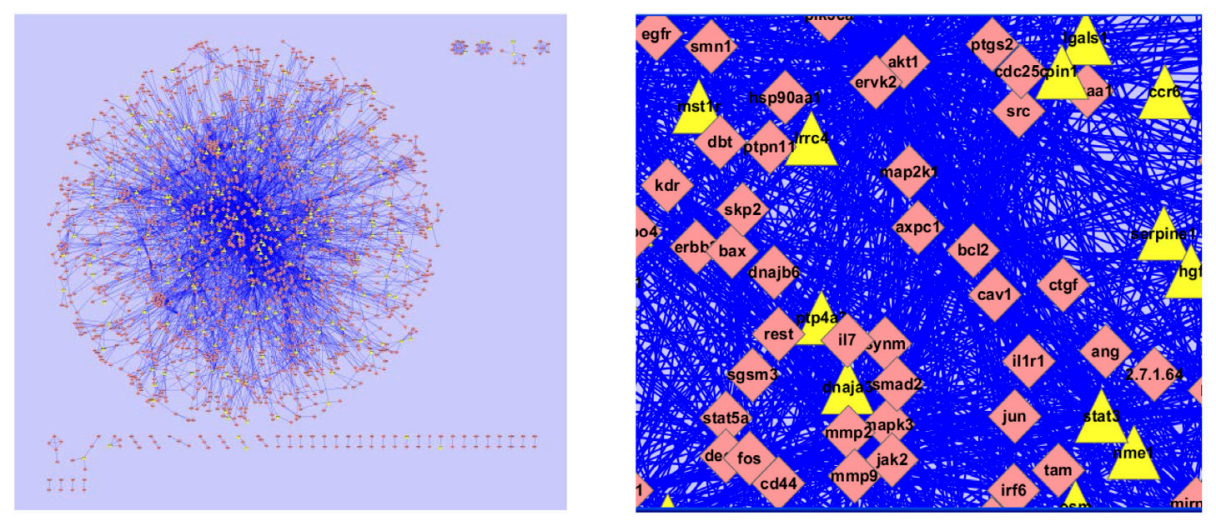

Figure 1. Network map of glioma protein interaction (overall + partial).

\section{Detection of molecular complexes}

Through MCOMD algorithm analysis, we identified 8 molecular complexes whose correlation integral values were higher than 3 (Figure 3).

\section{Molecular complex pathway enrichment}

The 8 names of protein molecule complexes were searched online to identify the relevant pathways (Table 2). Using hypergeometric distribution test software (DAVID) (Bader and Hogue, 2003) (parameters: count $=2$, EASE $=0.1$, "species and background" choosing "Homo sapiens"), we conducted function analysis of modules contained in the 2 networks. According to the pathway annotations, we identified biological signaling pathways (Ashburner et al., 2000) corresponding to the modules and sorted false-discovery rate values of biological processes, considering a false-discovery rate $\leq 1$ as a statistically significant difference in the biological process (Burkard et al., 2010). 


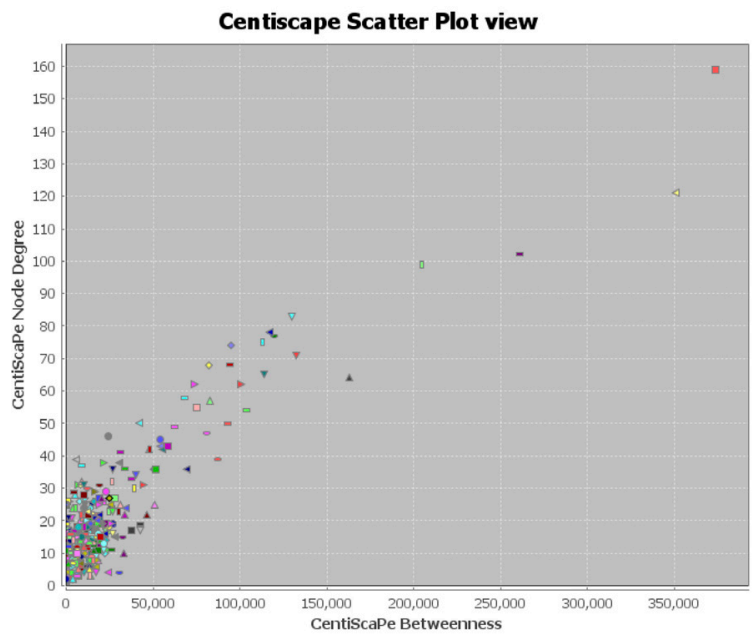

Figure 2. Connectivity degree of each node and betweenness (betweenness) comparison (horizontal axis represents betweenness, and the ordinate represents the connectivity degree. The graphic in the table represents each node in the network). The connectivity (number of nodes in the network) of nodes in the network obeys descending distribution, while the connectivity is greater than or equal to 50 , and the number of nodes corresponds to a sharp decrease.

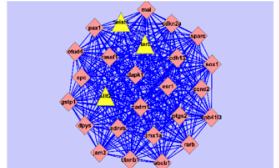

a: Complex 1 (relation score $=12.5$, 26 nodes and 325 edges).

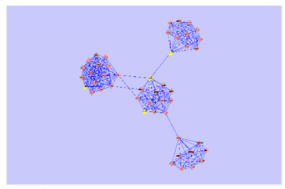

c: Complex 3 (relation score $=5.681$ 47 nodes and 267 edges).

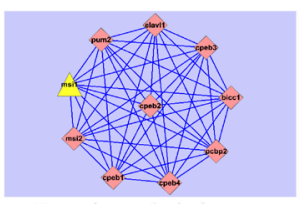

e: Complex 5 (relation score $=4.5$ 10 nodes and 45 edges)

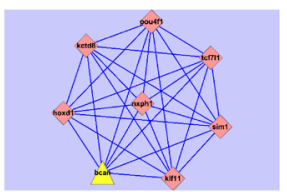

g: Complex 1 (relation score $=3.5$, nodes and 28 edges).

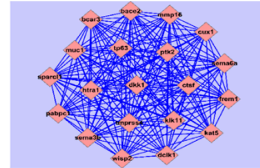

b: Complex 2 (relation score $=9.5$, 20 nodes and 190 edges)

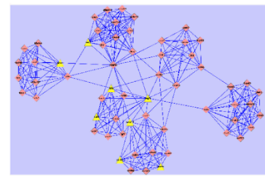

d: Complex 4 (relation score $=4.984$, 61 nodes and 304 edges)

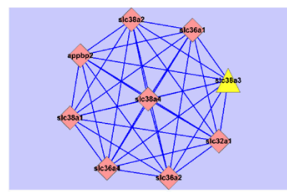

f: Complex 1 (relation score $=4,9$ nodes and 36 edges).

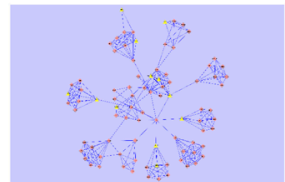

h: Complex 2 (relation score $=3.318$, 88 nodes and 292 edges)

Figure 3. Molecular complexes obtained by MCOMD algorithm analysis. 


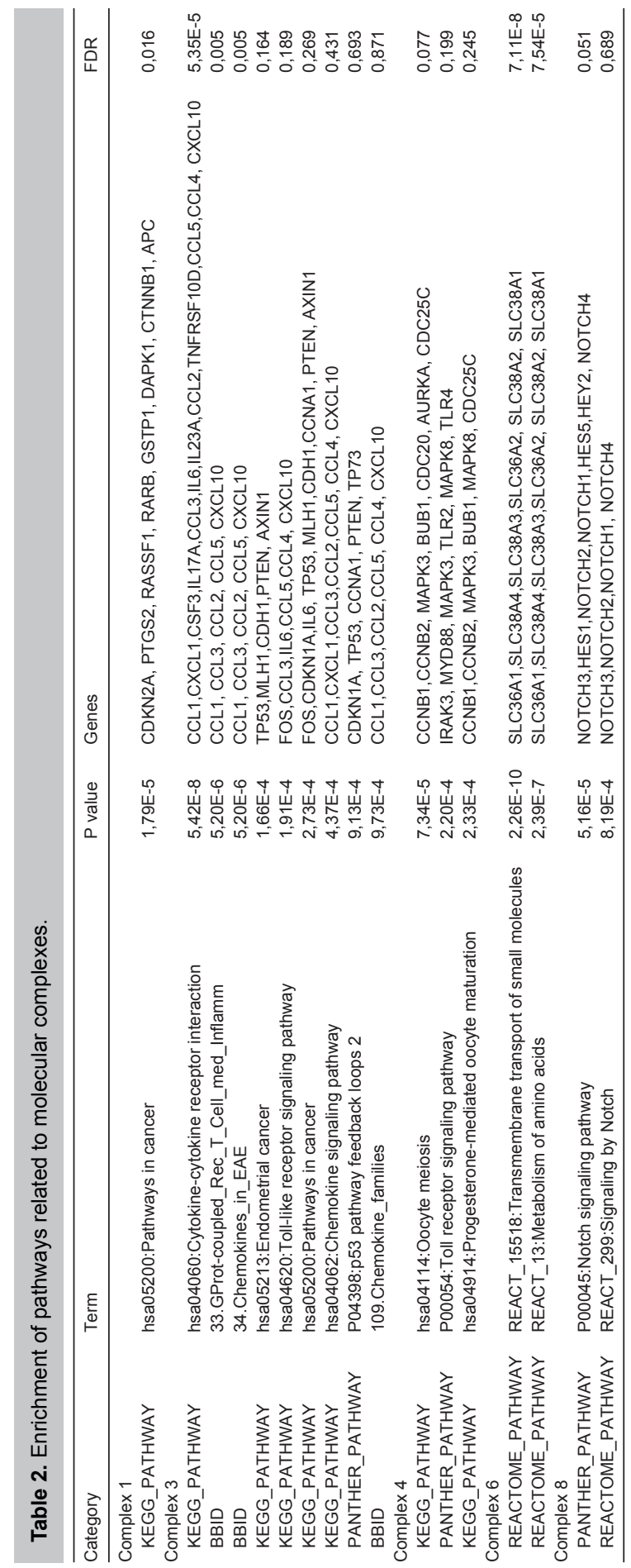




\section{DISCUSSION}

Based on the 378 genes identified by OMIM, we constructed a glioma protein interaction network containing 1814 nodes (proteins) with 1830 edges (interaction). We next examined whether the network could describe the molecular regulation of glioma development. According to the existing literature, the anti-apoptotic protein B-cell lymphoma 2 (BCL2) has been implicated in the pathogenesis of glioma. BCL2A1 is a potential biomarker that influences preoperative seizure occurrence and postoperative seizure control in patients with low-grade gliomas (You et al., 2013; Li et al., 2014). TP53 is a pivotal gene frequently mutated in diffuse gliomas and particularly in astrocytic tumors (Takami et al., 2014); CCL2 was among the first identified in gliomas, and it is overexpressed in colon carcinomas. Its silencing inhibits colon cancer cell proliferation or increases the sensitivity to apoptotic stimuli of glioma cells, suggesting an oncogenic role (Carrillode Sauvage et al., 2012). Increasing evidence suggests that interplay between the Wnt/ß-catenin and phosphoinositide 3-kinase /AKT signaling cascades are involved in tumor development and progression. Chen found that the expression levels of AKT1 in glioma cells were significantly correlated with the transcriptional activity of $\beta$-catenin (Pan et al., 2012). Mori found that the adenomatous polypopsis coli mutations in brain tumors were associated with the pathogenesis of one feature of Turcot syndrome (Siesjö et al., 1996); Zadeh et al., (2007) found that CDKN2Adeleted patients were younger than CDKN2A non-deleted patients in malignant gliomas in Iranian patients. Faulkner et al. found that neither epidermal growth factor receptor vIII (EGFRvIII) or EGFR were predictive of overall survival in their cohort; $49 \%$ of glioblastoma cases showed EGFR alterations, including 31\% with EGFRvIII, and thus EGFR and EGFRvIII can be used as therapeutic biomarkers of glioblastoma (Cherry and Stella, 2014). The microenvironment of glioblastoma contains high levels of inflammatory cytokine interleukin 6, which contributes to tumor progression and invasion (Gurgis et al., 2014). Among the factors and pathways implicated in glioma development and growth, the kinases phosphoinositide 3-kinase and mitogen-activated protein kinase are among the most studied (Daniel et al., 2014). Annibali et al., (2014) found that Myc inhibition reduces proliferation, increases apoptosis, and, remarkably, elicits the formation of multinucleated cells that then arrest or die by mitotic catastrophe, revealing a new role for Myc in the proficient division of glioma cells. Some results confirmed that PIK3CA mutations occurred in a significant number of human glioblastomas, making it a promising target for therapy, particularly for primary glioblastomas (Weber et al., 2011; Derakhshandeh-Peykar et al., 2012). A meta-analysis by Xiao et al. (2012) provided direct and strong evidences that mutations in the PTEN gene were correlated with the poor prognosis of glioma patients (Han et al., 2014). The PTGS2, EGFR, and various types of EGFR ligands are highly expressed in human gliomas and other cancers and are involved in tumor progression (Dancey, 2004). In gliomas, STAT3 can play tumor-suppressive or oncogenic roles depending on the tumor genetic background of the patient, but the target genes are largely unknown (Kruczyk et al., 2014).

These are relational pathogenesis of glioma. We constructed a network that comprises these genes or proteins The network appeared to be reliable and can be used to describe the interactions between molecules related to glioma.

Because the network is very large, we used the MCOMD algorithm to evaluate the network's regional integration using the correlation integral. The correlation integral describes proteins associated with the degree within the region. Proteins in the same molecular complexes generally have the same biological function, and thus unknown gene functions or new molecular functional 
groups can be identified. Eight molecular complexes showed correlation integrals of greater than 3. DAVID is not only extensive in gene annotation in different species, but also enriched with biological information for single genes. The protein molecule biological pathways of complexes 2 , 5 , and 7 are not existent, which may have two explanations. First, although the relevance of these molecular complex correlation integrals was higher, a protein with similar biological functions could not be confirmed. Second, existing studies have not revealed the biological pathways involved. Molecular complexes 1, 3, 4, 6, and8 were found to be involved many biological pathways. Table 2 shows its complexity, for which there was 1 biological pathway whose false-discovery rate $<1$ in molecular complex 1, 9 pathways in molecular complex 3, 3 pathways in molecular complex 4, 2 pathways in molecular complex 6 , and 2 in molecular complex 8.

Molecular complex 3 was predicted to be related to the cytokine-cytokine receptor interaction, Toll-like receptor signaling pathway, chemokine signaling pathway, p53 pathway feedback loops 2, and endometrial cancer. A previous study indicated that chemokine and chemokine receptor expression by tumor cells contributed to tumor growth and angiogenesis and thus these factors may be tumor markers and have crucial impacts on therapeutic interventions (Razmkhah et al., 2014).

There is increasing evidence that cytokines play roles in these processes. Cytokines directly influence the progression of malignant glioma, promoting or suppressing tumor progression (Zhou et al., 2014). Thus, the cytokine-cytokine receptor interaction pathway (CCL1, CXCL1, CCL3, CCL2, CCL5, CCL4, and CXCL10) and chemokine signaling pathway (CCL1, CXCL1, CSF3, IL17A, CCL3, IL6, IL23A, CCL2, TNFRSF10D, CCL5, CCL4, and CXCL10) require further analysis.

In addition to the other molecular complexes, progesterone-mediated oocyte maturation is related to the pathogenesis of glioma (Hassanzadeh and Arbabi, 2012); high notch pathway activation predicts a response to $y$ secretase inhibitors in the proneural subtype of glioma tumorinitiating cells (Saito et al., 2012). The genes involved in this signaling pathway may provide a basis for the molecular therapy to treat glioma. Glioma is not simply controlled by a particular gene or signaling pathway, but by a complex network system coordinately regulated and consisting of a variety of signaling pathways and multiple genes. In the signaling network, it is likely that there are some "key regulatory points".

Our study extended the original method used for glioma analysis from a single factor to a systematic, overall point perspective by constructing a network. Our results may provide new drug development guidance for treating glioma on the gene-protein network level. We used Cytoscape 2.82 for data mining and module analysis based on the OMIM database, and a small number of genes were identified because a single source of data was used, and because of the software features. Our constructed gene-protein interaction network did not reflect the regulatory relationship between the genes and proteins, and thus, further analysis is required.

\section{Conflicts of Interest}

The authors declare no conflict of interest.

\section{REFERENCES}

Amberger J, Bocchini CA, Scott AF and Hamosh A (2009). McKusick's Online Mendelian Inheritance in Man (OMIM). Nucleic Acids Res. 37: D793-D796.

Annibali D, Whitfield JR, Favuzzi E, Serrano E, et al. (2014). Myc inhibition is effective against glioma and reveals a role for 
Myc in proficient mitosis. Nat. Commun. 5: 4632.

Ashburner M, Ball CA, Blake JA, Botstein D, et al. (2000). Gene Ontology: tool for the unification of biology. The Gene Ontology Consortium. Nat. Genet. 25: 25-29.

Bader GD and Hogue CW (2003). An automated method for finding molecular complexes in large protein interaction networks. BMC Bioinformatics 4: 2.

Burkard TR, Rix U, Breitwieser FP, Superti-Furga G, et al. (2010). A computational approach to analyze the mechanism of action of the kinase inhibitor bafetinib. PLoS Comput. Biol. 18: e1001001.

Carrillo-de Sauvage MA, Gómez A, Ros CM, Ros-Bernal F, et al. (2012). CCL2-expressing astrocytes mediate the extravasation of T lymphocytes in the brain. Evidence from patients with glioma and experimental models in vivo. PLoS One 7: e30762.

Cherry AE and Stella N (2014). G protein-coupled receptors as oncogenic signals in glioma: Emerging therapeutic avenues. Neuroscience 14: 222-236.

Constantin A, Elkhaled A, Jalbert L, Srinivasan R, et al. (2012). Identifying malignant transformations in recurrent low grade gliomas using high resolution magic angle spinning spectroscopy. Artif. Intell. Med. 55: 61-70.

Dancey JE (2004). Predictive factors for epidermal growth factor receptor. Inhibitors-The bull's-eye hits the arrow. Cancer Cell 5: $411-415$

Daniel P, Filiz G, Brown DV, Hollande F, et al. (2014). Selective CREB-dependent cyclin expression mediated by the PI3K and MAPK pathways supports glioma cell proliferation. Oncogenesis 3: e108.

Dell'Albani P (2008). Stem cell markers in gliomas. Neurochem. Res. 33: 2407-2415.

Derakhshandeh-Peykar P, Alivi J, Hossein-nezhad A, Rautenstrauss B, et al. (2012). High frequency of mutations in the PIK3CA gene helical and kinase coding regions in a group of Iranian patients with high-grade glioblastomas: five novel mutations. J. Neurogenet. 25: 189-194.

Giacomelli L and Covani U (2010). Bioinformatics and data mining studies in oral genomics and proteomics: new trends and challenges. Open Dent. J. 4: 67-71.

Goodenberger ML and Jenkins RB (2012). Genetics of adult glioma. Cancer Genet. 205: 613-621.

Gurgis FM, Yeung YT, Tang MX, Heng B, et al. (2014). The p38-MK2-HuR pathway potentiates EGFRvIII-IL-1 $\beta$-driven IL-6 secretion in glioblastoma cells. Oncogene 10: 1038.

Hassanzadeh P and Arbabi E (2014). The effects of progesterone on glial cell line-derived neurotrophic factor secretion from C6 glioma cells. Iran J. Basic Med. Sci. 15: 1046-1052.

Huang DW, Sherman BT and Lempicki RA (2009). Systematic and integrative analysis of large gene lists using DAVID bioinformatics resources. Nat. Protoc. 4: 44-57.

Ideker T and Sharan R (2008). Protein networks in disease. Genome Res. 18: 644-652.

Kruczyk M, Przanowski P, Dabrowski M, Swiatek-Machado K, et al. (2014). Integration of genome-wide of Stat3 binding and epigenetic modification mapping with transcriptome reveals novel Stat3 target genes in glioma cells. Biochim. Biophys. Acta 14: 1341-1350.

Li W, Qian C, Wang L, Teng H, et al. (2013). Association of BCL2-938C>A genetic polymorphism with glioma risk in Chinese Han population. Tumour Biol. 35: 2259-2264.

Pan SJ, Zhan SK, Pei BG, Sun QF, et al. (2012). MicroRNA-149 inhibits proliferation and invasion of glioma cells via blockade of AKT1 signaling. Int. J. Immunopathol. Pharmacol. 25: 871-881.

Pospisil P, lyer LK, Adelstein SJ and Kassis Al (2006). A combined approach to data mining of textual and structured data to identify cancer-related targets. BMC Bioinformatics 7: 354.

Razmkhah M, Arabpour F, Taghipour M, Mehrafshan A, et al. (2014). Expression of chemokines and chemokine receptors in brain tumor tissue derived cells. Asian Pac. J. Cancer Prev. 15: 7201-7205.

Saito R, Smoot ME, Ono K, Ruscheinski J, et al. (2012). A travel guide to Cytoscape plugins. Nat. Methods 9: 1069-1076.

Strogatz SH (2001). Exploring complex networks. Nature 3: 268-276.

Shao W, Gu J, Huang C, Liu D, et al. (2014). Malignancy-associated metabolic profiling of human glioma cell lines using $1 \mathrm{H}$ NMR spectroscopy. Mol. Cancer 13: 197.

Shannon P, Markiel A, Ozier O, Baliga NS, et al. (2003). Cytoscape: a software environment for integrated models of biomolecular interaction networks. Genome Res. 13: 2498-2504.

Siesjö P, Visse E and Sjögren HO (1996). Cure of established, intracerebral rat gliomas induced by therapeutic immunizations with tumor cells and purified APC or adjuvant IFN-gamma treatment. J. Immunother. Emphasis Tumor Immunol. 19: 334-345.

Takami H, Yoshida A, Fukushima S, Arita H, et al. (2014). Revisiting TP5 mutations and immunohistochemistry-a comparative study in 157 diffuse gliomas. Brain Pathol. 10: 256-265.

Vailaya A, Bluvas P, Kincaid R, Kuchinsky A, et al. (2005). An architecture for biological information extraction and representation. Bioinformatics 21: 430-438. 
Weber GL, Parat MO, Binder ZA, Gallia GL, et al. (2011). Abrogation of PIK3CA or PIK3R1 reduces proliferation, migration, and invasion in glioblastoma multiforme cells. Oncotarget 2: 833-849.

Wen PY and Kesari S (2008). Malignant gliomas in adults. N. Engl. J. Med. 359: 492-507.

Xiao WZ, Han DH, Wang F, Wang YQ, et al. (2012). Relationships between PTEN gene mutations and prognosis in glioma: a meta-analysis. Tumour Biol. 35: 6687-6693.

Yang Y, Adelstein SJ and Kassis Al (2009). Target discovery from data mining approaches. Drug Discov. Today 2: 147-154.

You G, Feng L, Yan W, Zhang W, et al. (2013). BCL2A1 is a potential biomarker for postoperative seizure control in patients with low-grade gliomas. CNS Neurosci. Ther. 19: 882-888.

Zadeh MD, Amini R, Firoozray M and Derakhshandeh-Peykar P (2007). Frequent homozygous deletion of p16/CDKN2A gene in malignant gliomas of Iranian patients. Pak. J. Biol. Sci. 10: 4246-4250.

Zhao XM, Wang RS, Chen L and Aihara K (2008). Uncovering signal transduction networks from high-throughput data by integer linear programming. Nucleic Acids Res. 36: e48.

Zhou W, Jiang Z, Li X, Xu Y, et al. (2014). Cytokines: shifting the balance between glioma cells and tumor microenvironment after irradiation. J. Cancer Res. Clin. Oncol. 141: 575-589. 